\title{
Actively Biased $p$-Channel MOSFET Studied with Scanning Capacitance Microscopy
}

\author{
C.Y. Nakakura, ${ }^{\text {a) }}$ D.L. Hetherington, ${ }^{\text {a) }}$ M.R. Shaneyfelt, ${ }^{\text {a) }}$ P.E. Dodd, ${ }^{\text {a) }}$ and P. De Wolf \\ ${ }^{a}$ Microelectronics Development Laboratory, Sandia National Laboratories, Albuquerque, New Mexico 87185 \\ b)Digital Instruments, Santa Barbara, California 93117
}

\begin{abstract}
Scanning capacitance microscopy was used to study the cross section of an operating $p$-channel MOSFET. We discuss the novel test structure design and the modifications to the SCM hardware that enabled us to perform SCM while applying dc bias voltages to operate the device. The results are compared with device simulations performed with DAVINCI.

\section{Introduction}

As critical MOSFET dimensions have decreased, a need for high-resolution two-dimensional (2D) dopant profiling techniques has emerged. Scanning capacitance microscopy (SCM) has attracted considerable attention in response to this need because of its ability to measure 2D free carrier profiles with nanometer-scale resolution (1). While there is a large body of work using SCM to measure profiles of MOSFET devices, these studies have been limited to structures without independent electrical access to the device regions (source, drain, gate, and well), rendering them non-operational. In this work, we describe SCM measurements of a novel MOSFET test structure while gradually biasing the device from off to on. The evolution of the SCM images as a function of operating bias provides insight into changes in the channel region during MOSFET operation. The results are compared with device simulations of the free carrier concentration.
\end{abstract}

\section{Results and Discussion}

The sample used in this study is shown in Fig. 1. The die containing the test structure was mounted on a cut 16-pin dual in-line package (DIP) with bond wires connecting the transistor to the package leads [Fig. 1(a)]. The cross sections were polished with diamond-coated disks with incrementally decreasing particle size, followed by a final polish with commercial chemical mechanical polishing (CMP) slurries to obtain a finished surface with nominal RMS roughness on the angstrom level. The as drawn transistor width-to-length ratio (W/L) was $100 \mu \mathrm{m} / 0.6 \mu \mathrm{m}$ [Fig. 1(b)], minimizing the polish constraints by providing a greater distance to obtain the finished surface. The devices were fabricated at Sandia National Laboratories in a $5 \mathrm{~V}, 0.5 \mu \mathrm{m}$ (effective channel length) CMOS technology that uses shallow trench isolation (a) Bonding Wires Coated w/ Epoxy

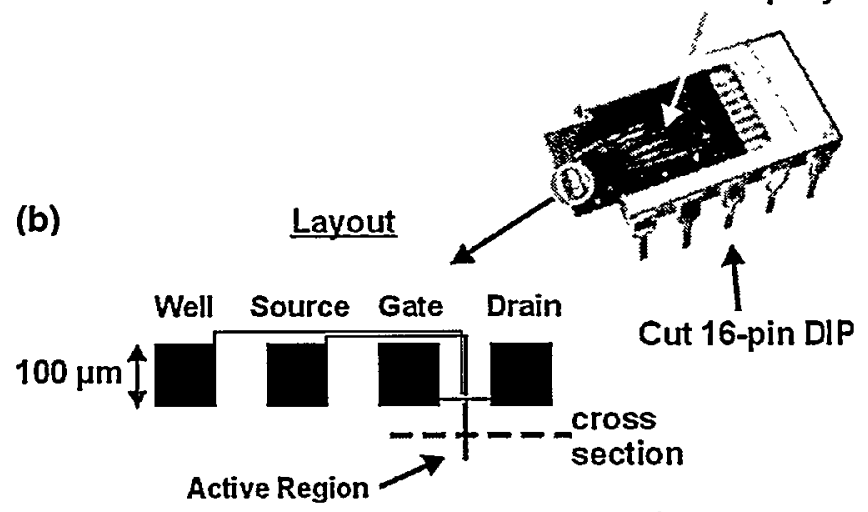

Fig. 1. (a) Photograph of mounted SCM sample. The die containing each MOSFET test structure is mounted hanging off the edge of the package to allow access to the cross-section for mechanical polishing. Bond wires connect the devices to the DIP leads and are coated in epoxy for protection while handling. (b) Drawing of the test structure layout. The location of the test structure is circled on the die in (a).

and CMP-planarization (2). The $p$-channel transistor was built in a retrograde $n$-well with a surface concentration of $\sim 3 \times 10^{17} \mathrm{~cm}^{-3}$ and has a $13 \mathrm{~nm}$ thermally grown gate oxide.

In SCM, an ac voltage is applied between the tip of an atomic force microscope (AFM) and a semiconducting sample, forming a small MOS capacitor. The ac bias voltage $(d V)$ induces a capacitance variation $(d C)$, which is measured using a high-frequency resonance circuit and is a direct measure of the local carrier concentration (3). In the standard SCM configuration, the tip is grounded while applying the ac bias to the entire sample, thus shorting out the device and prohibiting the use of dc bias voltages for MOSFET operation. In this study, the SCM hardware (Digital Instruments D5000 AFM with SCM capabilities) was modified to allow the application of the ac bias directly to the tip, as shown in the schematic diagram in Fig. 2. This permitted electrical access to the separate device regions so that dc bias voltages could be used to operate the transistor while simultaneously acquiring an SCM image. During SCM measurements, a model HP 4145 semiconductor parameter analyzer was used to supply the dc power for the device (Fig. 2), as well as to monitor the drain current. Measuring the drain 


\section{DISCLAIMER}

This report was prepared as an account of work sponsored by an agency of the United States Government. Neither the United States Government nor any agency thereof, nor any of their employees, make any warranty, express or implied, or assumes any legal liability or responsibility for the accuracy, completeness, or usefulness of any information, apparatus, product, or process disclosed, or represents that its use would not infringe privately owned rights. Reference herein to any specific commercial product, process, or service by trade name, trademark, manufacturer, or otherwise does not necessarily constitute or imply its endorsement, recommendation, or favoring by the United States Government or any agency thereof. The views and opinions of authors expressed herein do not necessarily state or reflect those of the United States Government or any agency thereof. 


\section{DISCLAIMER}

Portions of this document may be illegible in electronic image products. Images are produced from the best available original document. 


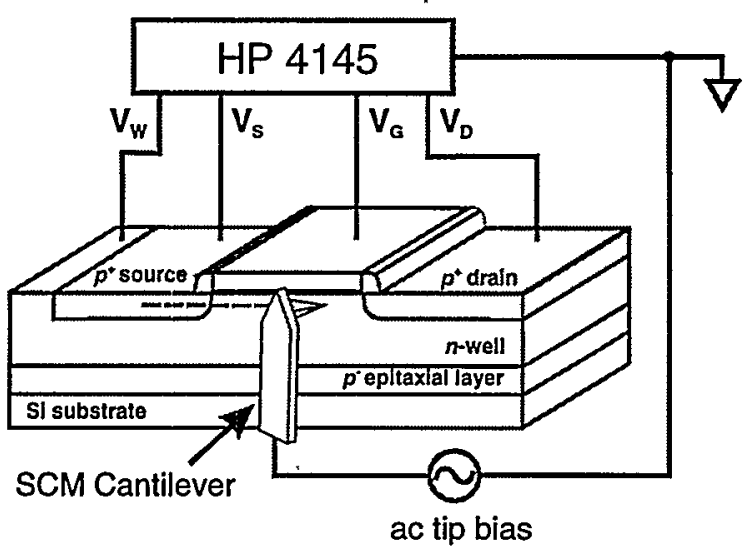

Fig. 2. Schematic of the SCM measurement showing a cross section layout of the PMOS device test structure used in this study.

current was critical to ensure that the SCM measurements were of a properly functioning device.

Fig. 3 shows simultaneously acquired (a) AFM and (b) SCM images of the cross-sectioned PMOS device. The topography of the device cross section is shown in the AFM image; the outline of the gate, as well as the boundary beneath the Ti- silicide, are clearly visible. Beneath the salicide, the implanted source and drain regions are revealed in the SCM image in Fig. 3(b). The SCM is very sensitive to charge defects in the sample, as seen near the curved bottom of the source.

To evaluate the impact of cross sectioning the device, current-voltage curves (drain current, $I_{D}$, vs. gate voltage, $V_{G}$ ) were recorded before and after polishing. We previously reported that the only change observed was an increase in leakage current, presumably due to conduction across the exposed edge of the device (4). However, the increase in leakage current can be minimized through careful polishing. This was demonstrated in a study where $I-V$ curves were recorded after each step in the polishing procedure, and the results are shown in Fig. 4. The plot shows the drain current
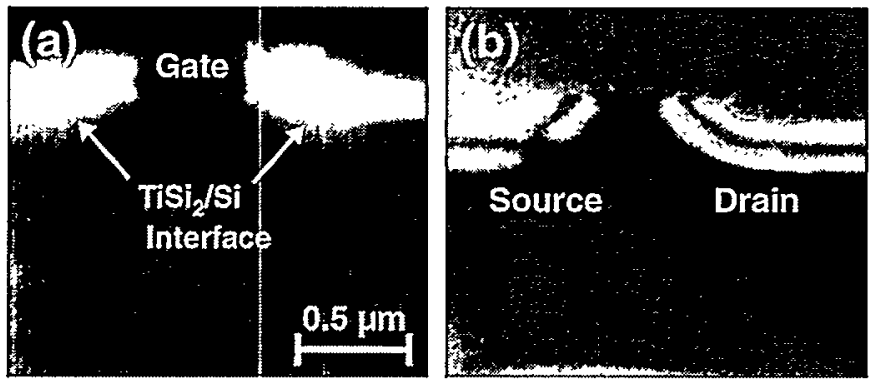

Fig. 3. (a) Topographical AFM image of the polished cross section of the PMOS device, and (b) the corresponding SCM image showing the $d C / d V$ response with drain, source, gate, and well at $V_{D}=-0.1 \mathrm{~V}, V_{G}=V_{S}=V_{W}=0$ $\mathrm{V}$, respectively (ac tip bias $=2.0 \mathrm{~V}$ ).

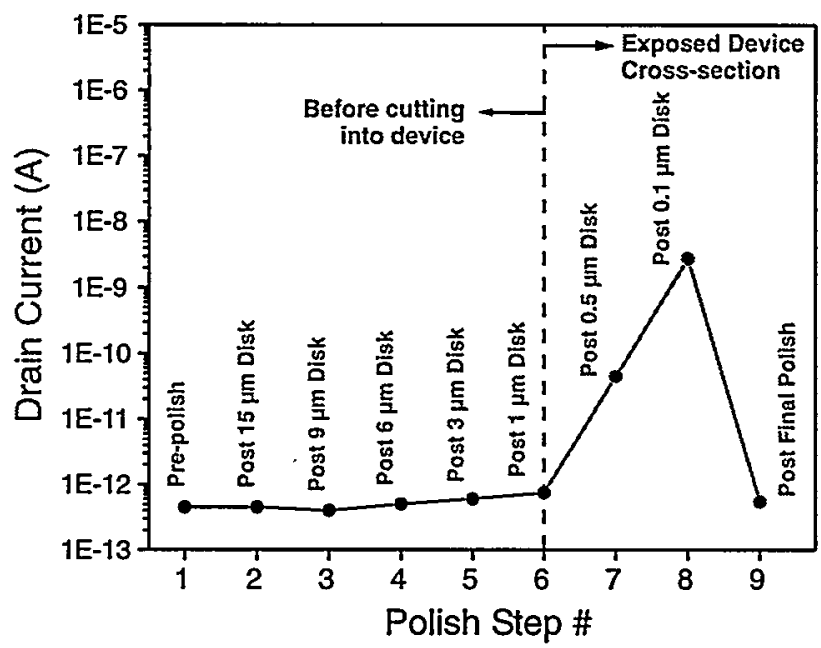

Fig. 4. Plot of drain current as a function of polish step during sample preparation of the PMOS device. The drain current was taken from $I-V$ curves (measured with $V_{D}=-0.1 \mathrm{~V} ; V_{S}=V_{W}=0 \mathrm{~V}$ ) at $V_{G}=1 \mathrm{~V}$.

at a gate voltage of $1 \mathrm{~V}$ (drain voltage, $V_{D}=-0.1 \mathrm{~V}$, and source and well voltages, $V_{S}=V_{W}=0 \mathrm{~V}$ ), where the device is off, as a function of polishing step. Starting with the prepolished sample (step \#1), the drain current remained in the sub-picoampere range after polishing with disks coated with particle sizes ranging from $15 \mu \mathrm{m}$ (step \#2) down to $1 \mu \mathrm{m}$ (step \#6). Once the cross section was exposed (steps \#7 and \#8), the current increased into the nanoampere range. This is the result of scratches in the device cross section, from the relatively large polishing particles, forming charge-trapping centers. Removing the scratches in the surface at the final polish step brought the drain current back down to the subpicoampere range. The only $I-V$ characteristic affected by cross sectioning was a small decrease in the drive current, which is proportional to $\mathrm{W} / \mathrm{L}$.

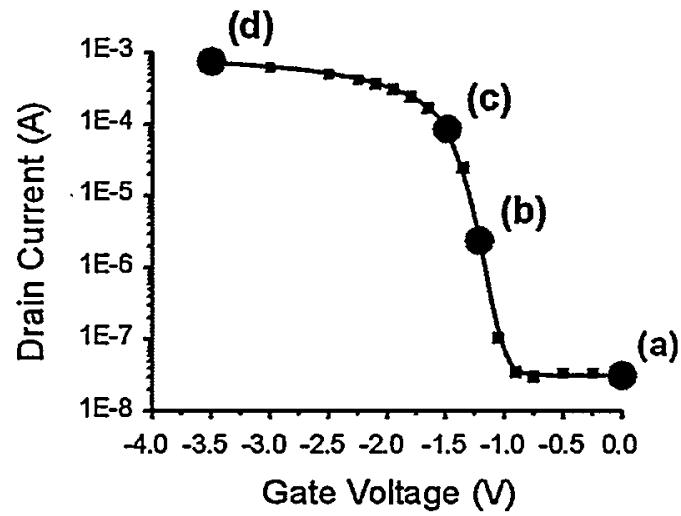

Fig. 5. $I_{D}$ vs. $V_{G}$ curve taken point-by-point during SCM image acquisition with $V_{D}=-0.1 \mathrm{~V}$. The circles denote where the SCM images in Fig. 6 were recorded. 

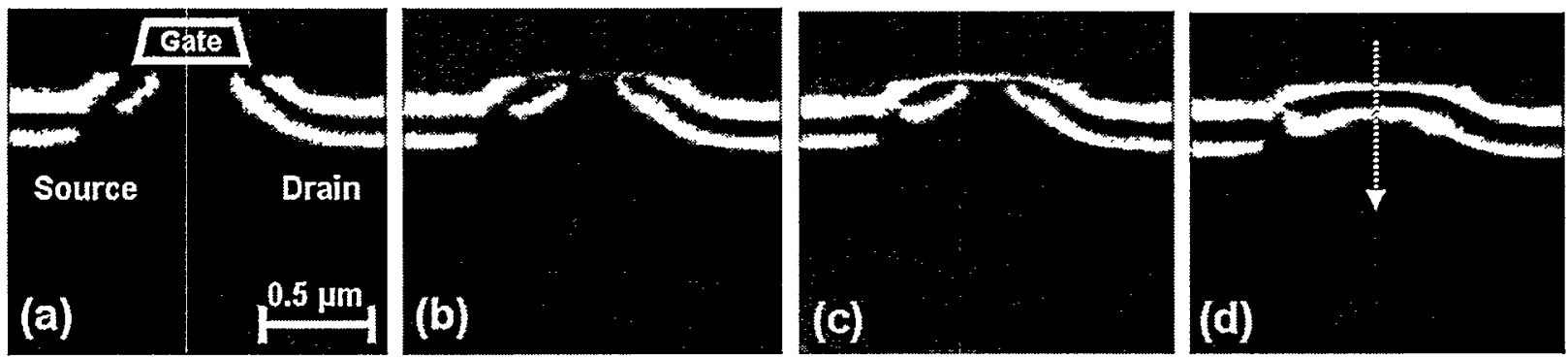

Fig. 6. Scanning capacitance microscopy images of the PMOS device recorded while maintaining constant voltages of $V_{D}=-0.1 \mathrm{~V}, V_{S}=0=V_{W}=0 \mathrm{~V}$, and sweeping the gate voltage as follows: (a) $0 \mathrm{~V}$, (b) $-1.2 \mathrm{~V}$, (c), $-1.5 \mathrm{~V}$, and (d) $-3.5 \mathrm{~V}$. The dashed line and arrow in (d) shows the location and direction of the line scans in Fig. 7.

In our earlier work, we performed SCM on a biased $p$ channel transistor with the drain held at $-3 \mathrm{~V}$ while sweeping the gate voltage (4). The analysis of the results was clouded by the interaction of the large drain bias with the ac signal applied to the tip. In this study, we have held the drain bias at $-0.1 \mathrm{~V}$ to minimize the impact on the results. The $I-V$ behavior of the sample was monitored in situ; the curve in Fig. 5 was taken point-by-point while acquiring a series of SCM images and shows $I-V$ behavior consistent with curves taken before running SCM. Note the high leakage current during this experiment $\left(3 \times 10^{-8} \mathrm{~A}\right.$ at $\left.V_{G}=0 \mathrm{~V}\right)$ compared with the data in Fig. $4\left(5 \times 10^{-13} \mathrm{~A}\right)$ for a freshly crosssectioned device. The leakage current has been consistently observed to increase after taking several SCM images. This is possibly the result of sample charging or local oxidation initiated by the biased tip while acquiring SCM images (5). Exposing cross-sectioned samples to air in the absence of a tip, for periods comparable to what a series of SCM experiments would take, did not show a significant increase in leakage current. We are currently performing studies to evaluate the impact of the leakage current on SCM images.

The sequence of SCM images in Fig. 6 corresponds to the locations on the $I-V$ curve of Fig. 5 denoted by the circles. At $V_{G}=0 \mathrm{~V}$ [Fig. 6(a)], the $d C / d V$ signal shows bright regions at the bottom of the source and drain. The lock-in detection operated in $\mathrm{R} / \theta$ mode, measuring the magnitude of the $d C / d V$ signal. Regions where the carrier concentration is low result in large changes in capacitance (high $d C / d V$ ) because carriers are depleted further into the sample for a given tip bias voltage. Thus, the bright regions at the bottom of the source and drain correspond to regions of low carrier concentration and are likely the result of the depletion regions. The second bright band just below the Ti-silicide, at the top of the source and drain, has been previously observed by others, but is not well-understood $(6,7)$. Increasing the gate voltage to (b) $-1.2 \mathrm{~V}$ and (c) $-1.5 \mathrm{~V}$ caused a spatial change in the $d C / d V$ signal beneath the gate region. A narrow band just beneath the gate can be seen linking the source and drain. In addition, the curved regions of the source and drain, which originally terminated perpendicular to the gate oxide, begin to lengthen towards one another. As seen in Fig. 5, these images were taken in the regime where the device was beginning to switch on, near the threshold voltage. Finally, by $-3.5 \mathrm{~V}$ the device was switched on (Fig. 5 ), and the region beneath the gate forms a continuous path of conduction between the source and drain, as shown in Fig. $6(d)$.

To help interpret the SCM images in Fig. 6, free carrier simulations were performed for the same biasing conditions using the device simulator, DAVINCI. Fig. 7 displays the (a)

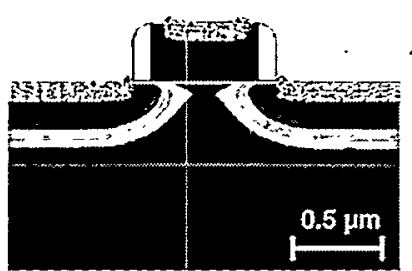

(b)

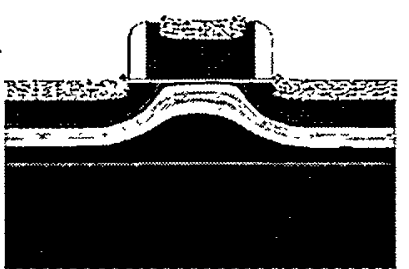

(c)

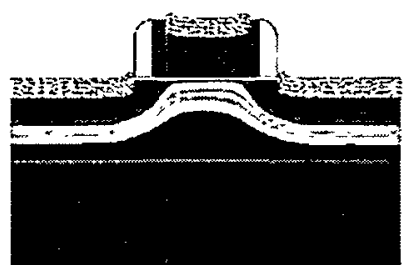

(d)

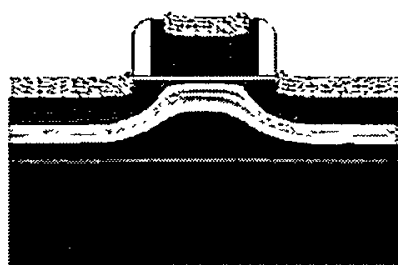

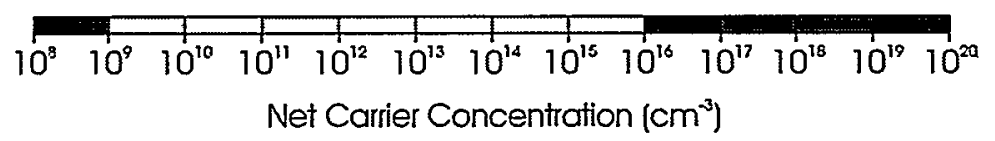

Fig. 7. Device simulations of a $0.5 \mu \mathrm{m}$-channel MOSFET using DAVINCI with $V_{D}=-0.1 \mathrm{~V}, V_{S}=V_{W}=0 \mathrm{~V}$, and varying the gate voltage as follows: (a) 0 $\mathrm{V}$, (b) $-1.2 \mathrm{~V}$, (c) $-1.5 \mathrm{~V}$, and (d) $-3.5 \mathrm{~V}$. The hatched regions correspond to Ti-silicide. 


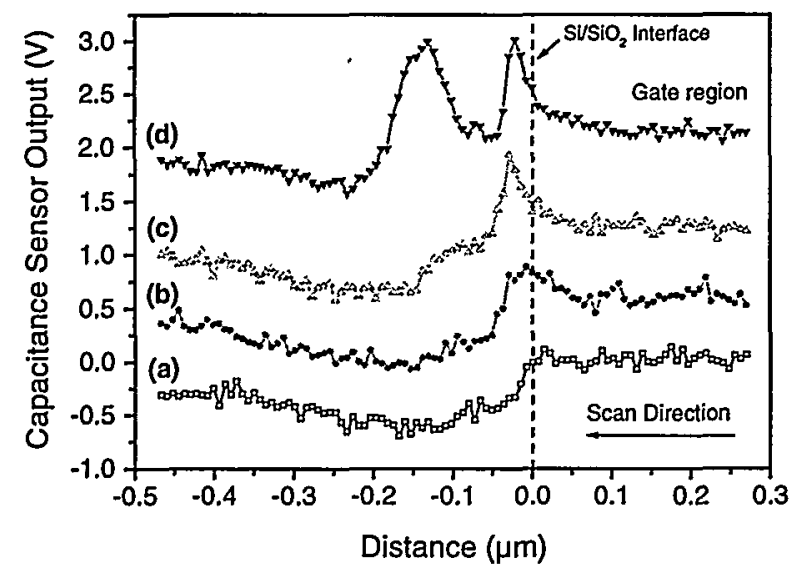

Fig. 8. Line profiles showing the capacitance sensor output along the line drawn through the channel, as shown in Fig. 6(d). The labeled curves were taken from the corresponding SCM images in Fig. $6\left[V_{D}=-0.1 \mathrm{~V}, V_{S}=0=\right.$ $V_{W}=0 \mathrm{~V}$, and sweeping the gate voltage: (a) $0 \mathrm{~V}$, (b) $-1.2 \mathrm{~V}$, (c), $-1.5 \mathrm{~V}$, and (d) $-3.5 \mathrm{~V}$. The dashed line delineates the interface between the $\mathrm{SiO}_{2}$ gate oxide and the $\mathrm{Si} n$-well.

simulation results for the $p$-channel transistor, plotting the net free carrier concentration in the device. Note that there is not a one-to-one relationship between the $d C / d V$ image produced by SCM and the simulated free carrier concentration, but the general characteristics are similar. The regions outlined by low carrier concentration in Fig. 7(a) show the same shape as the bottom bright bands in Fig. 6(a), delineating the zero-bias depletion regions. Under strong inversion conditions, the region of low net carrier concentration in Fig. 7(d) correlates well with the continuous band beneath the source and the drain [Fig. 6(d)]. Scanning capacitance microscopy images obtained for points operating near threshold [Figs. 6(b) and 6(c)], however, do not correlate well with device simulation, indicating possible interactions of the SCM tip with the surface. This discrepancy is not surprising, given that the simulations do not include the effect of the biased tip.

The changes in $d C / d V$ signal in the channel region of the SCM images from Fig. 6 are shown more clearly in Fig. 8. Each curve shows the sensor output along a line drawn through the channel, as shown in Fig. 6(d), for the SCM series in Fig. 6. The dashed line marks the location of the $\mathrm{SiO}_{2} / \mathrm{Si}$ interface, separating the gate oxide from the $n$-well, and is also the zero point reference in the distance scale on the horizontal axis. Curve (a) in Fig. 8 shows the $d C / d V$ response before the device was turned on, at a gate voltage of zero. As the gate voltage was increased to intermediate values, an increase in the sensor output just beneath the gate oxide was observed, resulting in the peak seen in curves (b) and (c) at $V_{G}=-1.2 \mathrm{~V}$ and $-1.5 \mathrm{~V}$, respectively. This peak was not observed at $V_{G}=0 \mathrm{~V}$ and, because the drain current began to increase under these conditions, it most likely indicates the presence of carriers in the channel region. The final curve (d) shows the channel region when the device was switched on; the second peak is from the depletion region spanning between source and drain, shown in Fig. 6(d).

\section{Summary}

We have begun to use SCM to study the dynamics of MOSFET operation by imaging the cross section of a biased $p$-channel device. By modifying the typical approach to SCM, we were able to observe the formation of a conducting path between the source and drain, demonstrating the promise of SCM as a method for studying device physics. We have also shown that exposing the cross section of a device does not have a severe impact on the $I-V$ characteristics, provided that the final polish step is done carefully. Free carrier simulations obtained using DAVINCI were able to capture some of the general features seen in the SCM measurements. However, simulations that address the three-dimensional interaction of the tip will be essential to fully interpret and quantify these measurements $(3,8)$.

This work was performed and supported by Sandia National Laboratories under DOE contract DE-AC04-94AL85000. Sandia National Laboratories is a multi-program laboratory operated by Sandia Corporation for the United States Department of Energy.

\section{References}

(1) J.J. Kopanski, J.F. Marchiando, D.W. Berning, R. Alvis, and H.E. Smith, J. Vac. Sci. Technol. B 16, 339 (1998).

(2) M. R. Shaneyfelt, P. E. Dodd, B. L. Draper, and R. S. Flores, IEEE Trans. Nucl. Sci. 45, 2584 (1998).

(3) M.L. O'Malley, G.L. Timp, W. Timp, S.V. Moccio, J.P. Gamo, and R.N. Kleiman, Appl. Phys. Lett. 74, 272 (1999).

(4) C.Y. Nakakura, D.L Hetherington, M.R. Shaneyfelt, P.J. Shea, and A.N. Erickson, Appl.. Phys. Lett., in press.

(5) P. Avouris, T. Hertel, and R. Martel, Appl. Phys. Lett. 71, 285 (1997).

(6) P. De Wolf, R. Stephenson, S. Biesemans, Ph. Jansen, G. Badenes, K. De Meyer, and W. Vandervorst, IEDM Tech. Dig (1998).

(7) H. Edwards, R. McGlothlin, R. San Martin, E. U, M. Gribelyuk, R. Mahaffy, C.K. Shih, R.S. List, and V.A. Ukrainstev, Appl. Phys. Lett. 72, 698 (1998).

(8) R.N. Kleiman, M.L O'Malley, F.H. Baumann, J.P. Garno, and G.L. Timp, IEDM Tech. Dig. (1997). 